\title{
Impulsivity, Sensation Seeking, and Risk-Taking Behaviors among HIV-Positive and HIV-Negative Heroin Dependent Persons
}

\author{
Koosha Paydary, ${ }^{1,2,3}$ Somayeh Mahin Torabi, ${ }^{2}$ SeyedAhmad SeyedAlinaghi, ${ }^{1}$ Mehri Noori, $^{2}$ \\ Alireza Noroozi, ${ }^{4,5}$ Sara Ameri, ${ }^{1,2,3}$ and Hamed Ekhtiari ${ }^{2,6}$ \\ ${ }^{1}$ Iranian Research Center for HIV/AIDS (IRCHA), Iranian Institute for Reduction of High-Risk Behaviors, \\ Tehran University of Medical Sciences (TUMS), Tehran, Iran \\ ${ }^{2}$ Neurocognitive Laboratory, Iranian National Center for Addiction Studies (INCAS), Tehran University of Medical Sciences (TUMS), \\ Tehran, Iran \\ ${ }^{3}$ Students' Scientific Research Center (SSRC), Tehran University of Medical Sciences (TUMS), Tehran, Iran \\ ${ }^{4}$ School of Advanced Technologies in Medicine (SATiM), Tehran University of Medical Sciences (TUMS), Tehran, Iran \\ ${ }^{5}$ Head of Substance Abuse Prevention and Treatment Office (SAPTO), Mental Health, Social Health and Addiction \\ Department (MeHSHAD), Ministry of Health and Medical Education (MoHME), Tehran, Iran \\ ${ }^{6}$ Translational Neuroscience Program, Institute for Cognitive Science Studies (ICSS), Tehran University of Medical Sciences (TUMS), \\ Tehran, Iran \\ Correspondence should be addressed to Hamed Ekhtiari; h_ekhtiari@razi.tums.ac.ir
}

Received 29 September 2015; Revised 11 January 2016; Accepted 31 January 2016

Academic Editor: Soraya Seedat

Copyright (C) 2016 Koosha Paydary et al. This is an open access article distributed under the Creative Commons Attribution License, which permits unrestricted use, distribution, and reproduction in any medium, provided the original work is properly cited.

Objective. The aim of this study was to compare impulsivity and risky decision making among HIV-positive and negative heroin dependent persons. Methods. We compared different dimensions of impulsivity and risky decision making in two groups of $60 \mathrm{HIV}$ positive and $60 \mathrm{HIV}$-negative male heroin dependent persons. Each group was comprised of equal numbers of current (treatment seeker) and former (abstinent) heroin addicts. Data collection tools included Balloon Analogue Risk Task (BART), Iowa Gambling Task (IGT), Barratt Impulsiveness Scale (BIS), and Zuckerman Sensation Seeking Scale (SSS). Results. In SSS, comprised of four subscales including thrill and adventure seeking (TAS), experience seeking (ES), disinhibition (DIS), and boredom susceptibility (BS), there was a borderline difference in DIS $(P=0.08)$ as HIV-positive group scored higher than HIV-negative group. Also, ES and total score were significantly higher among HIV-positive patients. In BART, HIV-positive subjects scored higher in risk taking than HIV-negative subjects as reflected in higher Average Number of puffs in Successful Balloons (ANSB). In BIS, HIV-positive group scored significantly higher in cognitive impulsivity $(\mathrm{CI})(P=0.03)$ and nonplanning impulsivity $(\mathrm{NPI})(P=0.05)$ in comparison to HIV-negative group. Also, current heroin addicts scored significantly higher in NPI compared to former addict HIV-negative participants $(P=0.015)$. IGT did not show any significant difference between groups. Conclusion. Higher levels of impulsivity and risk taking behaviors among HIV-positive heroin addicts will increase serious concerns regarding HIV transmission from this group to other opiate dependents and healthy people.

\section{Introduction}

Impulsive behavior is defined as behavior that is performed with little or inadequate forethought and may lead to the performance of risky activities [1]. The possible predisposing role of impulsivity and sensation seeking has been previously addressed in the acquisition of HIV infection worldwide $[2,3]$. On the other hand, psychological disorders and stigma in most of the People Living with HIV (PLWH) may lead to an increased rate of practicing impulsive and risky behaviors, that is, using nonsterile injection equipment and unprotected sexual activities [4]. Thus, the role of impulsive behavior 
is further highlighted with regard to the transmission of HIV infection from key populations at higher risk to the general population, especially in countries with concentrated epidemic [5].

The relationship between impulsivity and substance abuse is mutual; hence, substance abuse seems to be more prevalent among populations that score higher in impulsivity and sensation seeking measurement tools. In this regard, opiate abusers with higher impulsive behaviors are more likely to relapse after receiving appropriate treatments. Additionally, substance abuse may affect patients' cognitive and behavioral features. In fact, opiate abuse increases the practice of impulsive behavior which seems to be correlated with the severity of addiction. Additionally, impulsive drug users may show high levels of drug injection and unsterile needle use. Considering that using drugs through injection and unprotected sex are the major routes of HIV transmission, identification of the underlying psychological factors in HIV patients would be of utmost importance [6-8].

We conducted this study for the first time among Iranian PLWH to identify whether HIV infection is associated with impulsivity and sensation seeking as well as thrill and adventure seeking, experience seeking, boredom susceptibility, and nonplanning. Moreover, in order to elucidate the possible association of opiate addiction status (abstinence or active drug use) with impulsive behaviors, both HIV-positive and HIV-negative groups were comprised of equal numbers of current and former heroin addicts. Thus, the possible difference in risk taking, impulsivity rate, and sensation seeking between current and former heroin addicts could also be assessed.

\section{Materials and Methods}

2.1. Participants. This study was conducted among 120 participants who had a diagnosis of opiate dependence according to DSM-IV-TR criteria. The first group was comprised of 60 $\mathrm{HIV}$-positive males who were recruited in Iranian Research Center for HIV/AIDS (IRCHA), Tehran, Iran. The second group included $60 \mathrm{HIV}$-negative men that were recruited in the Iranian National Center of Addiction Studies (INCAS), Tehran, Iran. Active heroin users were recruited from Methadone Maintenance Programs' (MMP) waiting lists and their heroin use was confirmed with positive urine analysis for morphine and clinical interviews. Former heroin users were recruited from MMP with at least six months' participation and negative urine tests during last month. All the participants were recruited from September 2008 to September 2009 with simple sequential nonrandom sampling and were in the same demographic conditions. They were between 18 to 50 years old and provided informed written consent prior to participation. Also, the Institutional Review Board of Tehran University of Medical Sciences reviewed and approved the study protocol. All the participants of both groups were evaluated with Enzyme-Linked Immunosorbent Assay (ELISA) test for HIV infection and positive results were confirmed with subsequent Western blot. AIDS-related dementia, CD $4<500$ cells $/ \mu \mathrm{L}$, presence of any psychotic symptoms, diagnosis of dependence to other substances (except nico- tine), history of head trauma, and presence of any medical conditions, that is, liver, renal, cardiovascular, and neurological diseases, were considered as the exclusion criteria.

2.2. Measurements. All of participants were assessed by demographic forms and four questionnaires by a professionally trained interviewer under similar environmental conditions. The demographic questionnaire included questions about age, level of education, and condition of opiate addiction. All of the questionnaires were previously validated and attested in terms of reliability [9].

(1) The Farsi version of Zuckerman Sensation Seeking Scale (SSS) which defines the underlying reason for making risky decisions as the need for experiencing varied and novel situations [9]: this questionnaire has 40 items which examine the individual differences in four subscales including thrill and adventure seeking (TAS), experience seeking (ES), disinhibition (DIS), and boredom susceptibility (BS). Each item includes 10 questions and is scored $0-10$, while the sum of the scores would give the total score (0-40).

(2) The Farsi version of Balloon Analogue Risk Task (BART) test, which is a computerized model for measurement of risk-taking behavior [10]: in this test, participants are presented with 30 balloons on the computer screen which they may inflate by clicking the mouse button. Following each click, the balloon is either burst or further inflated, after which a specific amount of money is added to a temporary bank for that balloon. After each click, the participant may collect the temporary account at any point before the balloon bursts by clicking on a button marked as "collect" which would end the inflation of that balloon and add the temporary account to a safe account that is displayed on the screen. The more the eventual number of clicks for each balloon, the more money won from that balloon. The eventual size of balloons was variable and not predictable; hence, some balloons would reach the size of the screen while others would burst after several inflations. BART has a good correlation with the Iowa Gambling Task. The dependent measures of risk-taking in BART are defined as Average Number of puffs in Successful Balloons (ANSB), Number of Successful Balloons (NSB), and Average Number of puffs in Each Balloon (ANEB).

(3) The Farsi version of Barratt Impulsiveness Scale (BIS) which is a widely applied test for measurement of impulsivity [9]: the Farsi version of BIS used in this study was composed of 30 items on a four-point Likert scale focusing on the frequency of self-reported behavior (i.e., $1=$ never/rarely and $4=$ almost always) about three second-order factors including cognitive impulsivity (CI) (i.e., "I concentrate easily"), motor impulsivity (MI) (i.e., "I act on impulse"), and nonplanning impulsivity (NPI) (i.e., "I plan tasks carefully"). Thus, the items describe common impulsive behaviors and preferences about making decisions at special situations and thinking without relation to an exact time or location.

(4) The Farsi version of Iowa Gambling Task (IGT) in which participants are presented with four decks of cards labeled as A, B, C, and D [11]: for each deck of cards including 60 cards, a certain amount of reward and penalty has been considered. As the participant may see, the amount 
TABLE 1: Age, education, and duration of heroin addiction among HIV-positive and HIV-negative participants of the study.

\begin{tabular}{lccc}
\hline Variable $^{*}$ & HIV-positive group & HIV-negative group & $P$ value \\
\hline Age (years) & $36.43 \pm 7.65$ & $35.27 \pm 8.13$ & 0.42 \\
Education (years) & $9.83 \pm 2.56$ & $10.13 \pm 2.31$ & 0.50 \\
Heroin addiction duration (years) & $10.15 \pm 4.17$ & $9.64 \pm 6.57$ & 0.61 \\
\hline
\end{tabular}

* Data are represented as mean $\pm \mathrm{SD}$

Independent sample $t$-test.

of reward and penalties per chosen card from decks A and $B$ is more than cards from decks C and D. In this test, the participant should choose from four groups of decks in order to maximize the net amount of money won, while he/she is unaware of the total choices [12]. The net amounts of rewards and penalties are set in accordance with the fact that more choices from decks $\mathrm{C}$ and $\mathrm{D}$ would result in more amounts of total winning compared to the cards from decks A and B. After 100 choices are made, the total number of selections from advantageous decks (C and D) is subtracted from the number of selections from disadvantageous decks (A and B) and this difference is compared between the groups of study.

2.3. Statistical Analysis. Descriptive data were presented as mean $\pm \mathrm{SD}$ and frequency for quantitative data. After performing the Kolmogorov-smirnov test of normality, we performed independent sample $t$-test to compare quantitative variables between the two groups. In order to compare the mean scores between the four study subgroups, one-way ANOVA and post hoc analysis (Tukey's honest significance test) were used. Afterward, we performed analysis of covariance (ANCOVA) to exclude the influence of covariates on the mean of scores that were significantly different between four study subgroups. SPSS version 17.0 was used for data analysis and $P$ values equal to or less than 0.05 were considered statistically significant.

\section{Results}

3.1. Demographics and Substance Abuse History. One hundred and twenty male participants with the mean age of $38.5 \pm 7.88$ years and the educational average of $9.98 \pm 2.43$ years participated in this study. The total duration of opiate dependence was $9.90 \pm 5.48$ years. In the HIV-positive group, 31 participants were former heroin addicts and 29 of them were active heroin users. In the HIV-negative group, 30 participants were active heroin addicts and 30 were former addicts. Overall, 85 patients were crystalline heroin abusers (83 smokers and 2 injectors) among which 42 were HIVpositive and 42 were HIV-negative. Also, 35 patients were brown heroin abusers ( 29 heroin injectors, 3 heroin smokers, and 3 heroin sniffers) among which 18 were HIV-positive and 17 were HIV-negative. In the HIV-positive group, 17 patients were heroin injectors, while in the HIV-negative group, 12 patients were heroin injectors and 2 patients were crystalline heroin injectors. Based on analysis by age, education, and duration of addiction, there was no significant difference between the two groups of the study (Table 1).
3.2. The Zuckerman Sensation Seeking Scale (SSS). Results of the performed measurements are presented in Tables 2 and 3. There was no significant difference in TAS and BS between the groups (Table 2); however, there was a borderline difference in DIS, as HIV-positive group scored higher compared to HIV-negative group ( $P$ value $=0.083)$. In the total score of this test, HIV-positive group scored significantly higher than HIV-negative group $(P$ value $=0.03)$. Also, post hoc analysis showed that active opiate addict HIV-positive patients scored significantly higher than active opiate addict HIV-negative participants $(P$ value $=0.007)$. Also, ES was significantly higher in active opiate addict HIV-positive patients compared to former addict HIV-negative participants $(P$ value $=0.01)$. Additionally, HIV-positive patients with a positive history of Injection Drug Use (IDU) scored higher in ES ( $P$ value $=$ $0.04)$ and DIS $(P$ value $=0.01)$ compared to the HIV-negative group with a positive history of IDU, while the scores of TAS, BS, and total score were not significantly different between these two groups. In analysis of covariance of the ES score, result was correlated with the level of education $(P$ value $=$ 0.035). Even with the elimination of all factors, HIV infection had an effect on the ES scores $(P$ value $=0.008$, Table 4$)$.

3.3. The Balloon Analogue Risk Task (BART). HIV-positive group scored significantly higher than HIV-negative group regarding ANSB, NSB, and ANEB scores (Table 2). Also, analysis of the results between four groups showed significant difference too $(P$ value $=0.024$, Table 3$)$. According to post hoc analysis, former addict HIV-positive patients scored significantly higher than former addict HIV-negative participants in all three parameters $(P$ value $=0.015, P$ value $=0.001$, and $P$ value $=0.026$ for ANSB, NSB, and ANEB, respectively). However, ANSB and ANEB were not significantly different between HIV-positive patients with a history of IDU and HIV-negative patients with a history of IDU, while the difference in NSB was borderline significant $(P$ value $=0.07)$. There was no significant relationship between the items of this test and age, years of education, and addiction duration as demographic variables (Table 4).

3.4. The Barratt Impulsiveness Scale (BIS). After the examination of all three subscales between participants, HIV-positive group scored higher than HIV-negative group in CI and NPI (Table 2). Although this difference was significant for CI ( $P$ value $=0.035)$, it was borderline significant for NPI $(P$ value $=$ 0.053). Between four subgroups of the study, there was a significant difference in CI $(P$ value $=0.033)$ and NPI $(P$ value $=0.018$, Table 3$)$. Post hoc analysis showed that active 
TABLE 2: Comparison of subscale scores in each test between HIV-positive group and HIV-negative group.

\begin{tabular}{lcc}
\hline Item $^{*}$ & HIV-positive group & HIV-negative group \\
\hline & Zuckerman Sensation Seeking Scale (SSS) & $P$ value \\
Thrill and adventure seeking & $6.70 \pm 2.80$ & $6.33 \pm 2.55$ \\
Experience seeking & $4.28 \pm 1.90$ & $3.30 \pm 2.03$ \\
Disinhibition & $3.52 \pm 1.65$ & $2.95 \pm 1.89$ \\
Boredom susceptibility & $2.70 \pm 1.65$ & $2.48 \pm 1.44$ \\
SSS total score & $17.20 \pm 5.75$ & $15.07 \pm 5.13$ \\
\hline & Balloon Analogue Risk Task (BART) & 0.083 \\
Average Number of puffs in Successful Balloons & $34.58 \pm 17.11$ & 0.44 \\
Number of Successful Balloons & $17.52 \pm 4.02$ & $25.15 \pm 13.35$ \\
Average Number of puffs in Each Balloon & $30.40 \pm 12.40$ & $22.63 \pm 4.00$ \\
\hline & Barratt Impulsiveness Scale (BIS) & $23.64 \pm 11.58$ \\
Nonplanning impulsivity & $28.38 \pm 4.10$ & $26.75 \pm 4.99$ \\
Motor impulsivity & $24.43 \pm 5.04$ & $24.57 \pm 6.51$ \\
Cognitive impulsivity & $19.47 \pm 4.27$ & $17.67 \pm 4.94$ \\
BIS total score & $72.25 \pm 10.67$ & 6.002 \\
\hline & Iowa Gambling Task (IGT) & 0.004 \\
Total duration of IGT (seconds) & $286.54 \pm 168.38$ & 0.053 \\
Net amount of win $($ win - loss $)$ & $-12667.50 \pm-11223.32$ & 0.9 \\
IGT total score $[(C+D)-(A+B)]$ & $-2.56 \pm 21.54$ & $314.54 \pm 144.77$ \\
\hline
\end{tabular}

* Data are represented as mean \pm SD.

Independent sample $t$-test.

addict HIV-positive patients scored higher in NPI compared to former addict HIV-negative participants $(P$ value $=0.015)$. Also, former addict HIV-positive patients scored higher in CI compared to current addict HIV-negative group $(P$ value $=$ 0.020). Moreover, HIV-positive patients with a history of IDU scored higher compared to HIV-negative patients with a history of IDU in MI $(P$ value $=0.02)$ and total BIS score $(P$ value $=0.02)$, while the differences in NPI and CI were borderline significant $(P$ value $=0.07$ and $P$ value $=0.07$, respectively) . NPI had a significant relationship with age $(P$ value $=$ $0.014)$. CI was also significantly related to age $(P$ value $=$ $0.014)$, education $(P$ value $=0.003)$, and addiction duration $(P$ value $=0.015$, Table 4$)$.

3.5. The Iowa Gambling Task (IGT). There was no significant difference between HIV-positive group and HIV-negative group in three variables of total duration of IGT (seconds), net amount of win (win - loss), and IGT total score. Also the results did not reveal any significant difference among the four groups of the study. HIV-positive patients with a history of IDU did not score significantly different compared to HIVnegative patients with a history of IDU in any of the three variables of IGT.

\section{Discussion}

In the current study, we used multiple tests to assess the differences in impulsive behaviors, sensation seeking, and risk-taking among HIV-positive and negative heroin addicts. Hence, both groups were comprised of current and former addicts, and the relationships of current addiction status with each of the measured behavioral characteristics were also evaluated. According to our findings, HIV-positive opiate dependent persons reported higher levels of disinhibition in comparison with HIV-negative opiate dependent persons, regardless of addiction status, which implies that they may engage more in disinhibited behaviors that could enhance the risk of HIV transmission. Also, our results indicated that HIV patients may perform more risky behaviors due to their higher score in experience seeking which is in line with the findings of Hardy et al. [5]. As a matter of fact, HIV infection has reached concentrated epidemics among Iranian people who inject drugs; accordingly, the practice of impulsive behaviors and more experience seeking among this group (i.e., unprotected sexual intercourse) may further result in the spread of infection $[13,14]$. In line with this explanation, Gullette and Lyons declared that the scores of SSS are associated with the practice of risky behaviors such as unprotected sex with commercial sex workers, which may lead to the transmission of HIV epidemic from HIV-positive drug addicts to other key populations [15].

In our findings regarding comparison of BART, HIVpositive group scored higher than HIV-negative group in the three subscales which are known to be related to more risktaking behaviors. This is in line with previous reports that showed HIV infection is related to more risk-taking [16], although the definite influence of HIV infection on risktaking behavior remains unidentified. In fact, the current literature lacks enough evidence to support the idea that the incidence of HIV infection among opiate users with more risk-taking behavior is more compared to opiate users who have scored less in such tests. Additionally, modeling of 
TABLE 3: Comparison of subscale scores in each test between current and former opiate addict HIV-positive and HIV-negative participants.

\begin{tabular}{|c|c|c|c|c|c|}
\hline \multirow{2}{*}{ Item $^{*}$} & \multicolumn{2}{|c|}{ HIV-positive group } & \multicolumn{2}{|c|}{ HIV-negative group } & \multirow{2}{*}{$P$ value } \\
\hline & Current addicts & Former addicts & Current addicts & Former addicts & \\
\hline \multicolumn{6}{|c|}{ Zuckerman Sensation Seeking Scale (SSS) } \\
\hline $\begin{array}{l}\text { Thrill and adventure } \\
\text { seeking }\end{array}$ & $6.41 \pm 2.99$ & $6.97 \pm 2.63$ & $6.33 \pm 2.18$ & $6.33 \pm 2.91$ & 0.75 \\
\hline Experience seeking & $4.93 \pm 2.08$ & $3.68 \pm 1.51$ & $3.27 \pm 1.72$ & $3.33 \pm 2.33$ & 0.004 \\
\hline Disinhibition & $3.72 \pm 1.85$ & $3.32 \pm 1.44$ & $2.77 \pm 1.99$ & $3.13 \pm 1.79$ & 0.22 \\
\hline Boredom susceptibility & $3.07 \pm 1.79$ & $2.35 \pm 1.45$ & $2.73 \pm 1.23$ & $2.23 \pm 1.61$ & 0.14 \\
\hline SSS total score & $18.14 \pm 6.31$ & $16.32 \pm 5.12$ & $15.10 \pm 4.59$ & $15.03 \pm 5.70$ & 0.10 \\
\hline \multicolumn{6}{|c|}{ Balloon Analogue Risk Task (BART) } \\
\hline $\begin{array}{l}\text { Average Number of puffs } \\
\text { in Successful Balloons }\end{array}$ & $33.00 \pm 13.57$ & $35.93 \pm 19.80$ & $26.66 \pm 14.78$ & $23.70 \pm 11.88$ & 0.012 \\
\hline $\begin{array}{l}\text { Number of Successful } \\
\text { Balloons }\end{array}$ & $17.63 \pm 3.63$ & $17.43 \pm 4.40$ & $22.21 \pm 4.25$ & $23.03 \pm 3.77$ & $<0.001$ \\
\hline $\begin{array}{l}\text { Average Number of puffs } \\
\text { in Each Balloon }\end{array}$ & $29.21 \pm 10.12$ & $31.43 \pm 14.18$ & $24.93 \pm 12.82$ & $22.40 \pm 10.32$ & 0.02 \\
\hline \multicolumn{6}{|c|}{ Barratt Impulsiveness Scale (BIS) } \\
\hline Nonplanning impulsivity & $29.83 \pm 4.08$ & $27.03 \pm 3.70$ & $27.23 \pm 5.57$ & $26.27 \pm 4.38$ & 0.018 \\
\hline Motor impulsivity & $27.07 \pm 4.58$ & $23.84 \pm 5.45$ & $25.30 \pm 6.30$ & $23.83 \pm 6.84$ & 0.64 \\
\hline Cognitive impulsivity & $20.59 \pm 4.13$ & $18.42 \pm 4.19$ & $17.07 \pm 4.09$ & $18.27 \pm 5.68$ & 0.033 \\
\hline BIS total score & $75.41 \pm 9.77$ & $69.29 \pm 10.77$ & $69.60 \pm 13.45$ & $68.57 \pm 14.79$ & 0.13 \\
\hline \multicolumn{6}{|c|}{ Iowa Gambling Task (IGT) } \\
\hline $\begin{array}{l}\text { Total duration of IGT } \\
\text { (seconds) }\end{array}$ & $268473.43 \pm 104624.35$ & $301947.19 \pm 208890.0$ & $333373.52 \pm 185294.59$ & $296714.35 \pm 88304$ & 0.58 \\
\hline $\begin{array}{l}\text { Net amount of win (win } \\
- \text { loss) }\end{array}$ & $-13141.30 \pm 10152.75$ & $-12263.89 \pm 12239.43$ & $-14526.09 \pm 12693.19$ & $-13959.78 \pm 16297$ & 0.93 \\
\hline $\begin{array}{l}\text { IGT total score } \\
{[(C+D)-(A+B)]}\end{array}$ & $-3.48 \pm 18.29$ & $-1.78 \pm 24.30$ & $-5.57 \pm 26.47$ & $-5.74 \pm 32.63$ & 0.94 \\
\hline
\end{tabular}

${ }^{*}$ Data are represented as mean \pm SD.

One-way ANOVA.

the participants' behavior in BART has revealed that poor performance in heroin abusers is probably due to rewarddependency and insensitivity to evaluation $[16,17]$. Furthermore, the results of BIS in our survey showed that HIV infected patients scored higher with regard to nonplanning impulsivity and cognitive impulsivity in comparison with HIV-negative group.

With regard to opiate addiction, the differences between active drug users and former users engaging in impulsive behaviors corroborate the result of Passetti et al. [18]. Their finding was related to the fact that addiction can influence decision-making and increase the performance of impulsive behaviors [19-21]. Previously, Stout et al. used cognitive modeling to study decision-making deficits in cocaineabusers. Their study showed that cocaine-abusers have poor performance in IGT [22]. In fact, HIV infected substance users may have impaired performance on measures that involve the prefrontal-subcortical systems such as the IGT [23]. In our analysis, however, there was no significant difference in IGT results between HIV-positive group and HIV-negative group. Such observed differences could be explained by the different social settings as well as the diversity of attitudes and knowledge about stigmatization toward addiction and HIV infection between societies [24, 25]. The absence of group differences in IGT in our study may also be explained by the fact that there are different behavioral traits of impulsivity. Thereby, although we were not able to identify a significant difference in the results of IGT concerning cognitive impulsivity, the HIV infected patients scored higher in the cognitive impulsivity subscale measured by BIS. This finding may indicate a higher level of self-reported cognitive impulsivity compared to impulsive decision-making among HIV-positive patients. Although the relevance of observed differences in the obtained scores of self-reported cognitive impulsivity remains unidentified in this study, it may be related to the high levels of perceived stigma among Iranian PLWH [26].

This study has several limitations: first, we did not assess the effect of self-reported risky behaviors such as injection history, risky and unprotected sexual behaviors, aggression, and history of imprisonment on the measured items. Also, we were unable to collect data regarding the route of HIV acquisition and duration of infection among HIV-positive group. Another limitation was that all of our participants were male subjects. The sample size of our study was relatively small. For future studies, we suggest investigation of the risk-taking and 
TABLE 4: Models of the covariance analysis (ANCOVA) for exclusion of the influence of covariates were obtained for experience seeking domain of the sensation seeking scale, variables of the balloon analogue risk task (Average Number of puffs in Successful Balloons, Number of Successful Balloons, and Average Number of puffs in Each Balloon), and the nonplanning and cognitive impulsivity from Barratt Impulsiveness Scale.

\begin{tabular}{|c|c|c|c|c|c|c|c|c|}
\hline & $\begin{array}{l}\text { Corrected } \\
\text { model }\end{array}$ & Intercept & Age (years) & $\begin{array}{l}\text { Education } \\
\text { (years) }\end{array}$ & $\begin{array}{c}\text { Opiate } \\
\text { dependence } \\
\text { duration } \\
\text { (years) }\end{array}$ & $\begin{array}{c}\text { HIV } \\
\text { infection }\end{array}$ & $\begin{array}{c}\text { Opiate } \\
\text { dependence } \\
\text { status } \\
\text { (former } \\
\text { versus } \\
\text { current) }\end{array}$ & $\begin{array}{l}\text { Receiving } \\
\text { HIV } \\
\text { treatment }\end{array}$ \\
\hline \multicolumn{9}{|c|}{ Experience seeking } \\
\hline Mean square & 14.27 & 107.10 & 7.22 & 16.25 & 11.00 & 25.83 & 5.53 & 7.69 \\
\hline$F$ & 3.98 & 29.87 & 2.01 & 4.53 & 3.06 & 7.20 & 1.54 & 2.14 \\
\hline$P$ value & 0.001 & $<0.01$ & 0.159 & 0.35 & 0.083 & 0.008 & 0.217 & 0.146 \\
\hline \multicolumn{9}{|c|}{ Average Number of puffs in Successful Balloons } \\
\hline Mean square & 527.04 & 4193.4 & 174.28 & 15.69 & 47.10 & 2487.03 & 14.19 & 179.10 \\
\hline$F$ & 2.21 & 17.58 & 0.731 & 0.066 & 0.197 & 10.42 & 0.018 & 0.751 \\
\hline$P$ value & 0.048 & $<0.01$ & 0.395 & 0.798 & 0.658 & 0.002 & 0.895 & 0.388 \\
\hline \multicolumn{9}{|c|}{ Number of Successful Balloons } \\
\hline Mean square & 126.94 & 847.47 & 1.09 & 5.28 & 15.91 & 720.09 & 1.21 & 2.75 \\
\hline$F$ & 7.63 & 50.95 & 0.066 & 0.318 & 0.957 & 43.29 & 0.73 & 0.165 \\
\hline$P$ value & $<0.01$ & $<0.01$ & 0.798 & 0.574 & 0.330 & $<0.01$ & 0.787 & 0.685 \\
\hline \multicolumn{9}{|c|}{ Average Number of puffs in Each Balloon } \\
\hline Mean square & 281.03 & 3064.51 & 153.83 & 0.058 & 4.14 & 1284.11 & 0.448 & 135.18 \\
\hline$F$ & 1.90 & 20.74 & 1.04 & $<0.01$ & 0.028 & 8.69 & 0.003 & 0.915 \\
\hline$P$ value & 0.87 & $<0.01$ & 0.310 & 0.984 & 0.867 & 0.004 & 0.956 & 0.341 \\
\hline \multicolumn{9}{|c|}{ Nonplanning impulsivity } \\
\hline Mean square & 68.76 & 3533.44 & 118.46 & 55.24 & 29.42 & 91.09 & 75.97 & 11.19 \\
\hline$F$ & 3.64 & 187.10 & 6.27 & 2.92 & 1.55 & 4.82 & 4.02 & 0.593 \\
\hline$P$ value & 0.002 & $<0.01$ & 0.014 & 0.09 & 0.215 & 0.03 & 0.047 & 0.443 \\
\hline \multicolumn{9}{|c|}{ Cognitive impulsivity } \\
\hline Mean square & 93.89 & 2113.70 & 114.29 & 172.64 & 112.28 & 86.46 & 0.006 & 40.24 \\
\hline$F$ & 5.13 & 115.58 & 6.25 & 9.44 & 6.14 & 4.72 & $<0.01$ & 2.20 \\
\hline$P$ value & $<0.01$ & $<0.01$ & 0.014 & 0.003 & 0.015 & 0.032 & 0.986 & 0.141 \\
\hline
\end{tabular}

impulsivity among HIV-positive active drug users in female subjects and other key populations, that is, sex workers.

In conclusion, heroin dependent PLWH of this study scored significantly higher than HIV-negative group on different risk taking, impulsivity, and sensation seeking dimensions, which may render them susceptible to the practice of more risky behaviors. Such risky behaviors may further decrease the adherence to antiretroviral therapy and negatively impact their social relationships. Another consequence of practicing risky behaviors among opiate dependent HIV patients is the possible contribution of such behaviors to the spread of HIV to the general public. Thereby, interventions such as behavioral educations or psychosocial rehabilitations should focus on decreasing the practice of impulsive behaviors among this group of patients. Since the prevalence of psychiatric disorders is quite high among PLWH $[27,28]$, the timely diagnosis of substance abuse and concomitant psychological impairments for performing interventions that would halt the progress of perceived stigmatization is of great significance. Also, teaching PLWH to use coping strategies to deal with HIV stigma may lead to the improvement of the overall psychological well-being [29]. Considering that the main route of HIV acquisition in Iran is using drug and that current heroin addicts in both groups scored higher in NPI compared to former addicts, preventive and therapeutic interventions aiming at opiate addiction may further decrease the rate of HIV transmission in Iran.

\section{Conflict of Interests}

The authors declare that there is no conflict of interests regarding the publication of this paper.

\section{Acknowledgments}

The authors would like to thank all the staff at Iranian Research Center for HIV/AIDS (IRCHA) and Iranian National Center for Addiction Studies (INCAS). This study 
was supported by Tehran University of Medical Sciences grant provided to Dr. Hamed Ekhtiari at INCAS.

\section{References}

[1] R. F. Leeman and M. N. Potenza, "Similarities and differences between pathological gambling and substance use disorders: a focus on impulsivity and compulsivity," Psychopharmacology, vol. 219, no. 2, pp. 469-490, 2012.

[2] A. J. Stewart, C. Theodore-Oklota, W. Hadley, L. K. Brown, G. Donenberg, and R. DiClemente, "Mania symptoms and HIVrisk behavior among adolescents in mental health treatment," Journal of Clinical Child \& Adolescent Psychology, vol. 41, no. 6, pp. 803-810, 2012.

[3] J. Hayaki, B. J. Anderson, and M. D. Stein, "Sexual risk-taking mediates the association between impulsivity and acquisition of sexually transmitted infections among hazardously drinking incarcerated women," The American Journal on Addictions, vol. 21, supplement 1, pp. S63-S71, 2012.

[4] P. Razavi, K. Hajifathalian, B. Saeidi et al., "Quality of life among persons with HIV/AIDS in Iran: internal reliability and validity of an international instrument and associated factors," AIDS Research and Treatment, vol. 2012, Article ID 849406, 6 pages, 2012.

[5] D. J. Hardy, S. A. Castellon, C. H. Hinkin, A. J. Levine, and M. N. Lam, "Sensation seeking and visual selective attention in adults with HIV/AIDS," AIDS and Behavior, vol. 12, no. 6, pp. 930-934, 2008.

[6] J. L. Grenard, S. L. Ames, and A. W. Stacy, "Deliberative and spontaneous cognitive processes associated with HIV risk behavior," Journal of Behavioral Medicine, vol. 36, no. 1, pp. 95107, 2013.

[7] P. T. Harrell, B. E. Mancha, H. Petras, R. C. Trenz, and W. W. Latimer, "Latent classes of heroin and cocaine users predict unique HIV/HCV risk factors," Drug and Alcohol Dependence, vol. 122, no. 3, pp. 220-227, 2012.

[8] D. A. Nielsen, A. Ho, A. Bahl et al., "Former heroin addicts with or without a history of cocaine dependence are more impulsive than controls," Drug and Alcohol Dependence, vol. 124, no. 1-2, pp. 113-120, 2012.

[9] H. Ekhtiari, H. Safaei, G. Esmaeeli Djavid, M. K. Atefvahid, H. Edalati, and A. Mokri, "Reliability and validity of persian versions of eysenck, barratt, dickman and zuckerman questionnaires in assessing risky and impulsive behaviors," Iranian Journal of Psychiatry and Clinical Psychology, vol. 14, no. 3, pp. 326-336, 2008.

[10] H. Ekhtiari, A. Behzadi, and A. Mokri, "Computerized persian version of balloon analogue risk task, an assessment methods for risk taking tendencies," New Advances in Cognitive Sciences, vol. 3, no. 1, pp. 28-36, 2003.

[11] H. Ekhtiari, A. Behzadi, A. Jannati, and A. Mokri, "Cultural characteristics in standard and variant persian versions of iowa gambling task," New Advances in Cognitive Sciences, vol. 3, no. 1, pp. 46-57, 2002.

[12] D. G. Smith, L. Xiao, and A. Bechara, "Decision making in children and adolescents: impaired iowa gambling task performance in early adolescence," Developmental Psychology, vol. 48, no. 4, pp. 1180-1187, 2012.

[13] A. Mirzazadeh, A. A. Haghdoost, S. Nedjat, S. Navadeh, W. McFarland, and K. Mohammad, "Accuracy of HIV-related risk behaviors reported by female sex workers, Iran: a method to quantify measurement bias in marginalized populations," AIDS and Behavior, vol. 17, no. 2, pp. 623-631, 2013.

[14] M. Malekinejad, M. Mohraz, N. Razani et al., "High HIV prevalence in a respondent-driven sampling survey of injection drug users in Tehran, Iran," AIDS and Behavior, vol. 19, no. 3, pp. 440-449, 2014.

[15] D. L. Gullette and M. A. Lyons, "Sensation seeking, self-esteem, and unprotected sex in college students," The Journal of the Association of Nurses in AIDS Care, vol. 17, no. 5, pp. 23-31, 2006.

[16] R. Charnigo, S. M. Noar, C. Garnett, R. Crosby, P. Palmgreen, and R. S. Zimmerman, "Sensation seeking and impulsivity: combined associations with risky sexual behavior in a large sample of young adults," Journal of Sex Research, vol. 50, no. 5, pp. 480-488, 2013.

[17] P.-W. Wang, H.-C. Lin, H.-C. Wu et al., "Explicit and implicit heroin-related cognitions and heroin use among patients receiving methadone maintenance treatment," Comprehensive Psychiatry, vol. 56, pp. 155-160, 2015.

[18] F. Passetti, L. Clark, M. A. Mehta, E. Joyce, and M. King, "Neuropsychological predictors of clinical outcome in opiate addiction," Drug and Alcohol Dependence, vol. 94, no. 1-3, pp. 82-91, 2008.

[19] M. Boog, A. E. Goudriaan, B. J. M. V. D. Wetering, M. Polak, H. Deuss, and I. H. A. Franken, "Rash Impulsiveness and Reward Sensitivity as predictors of treatment outcome in male substance dependent patients," Addictive Behaviors, vol. 39, no. 11, pp. 1670-1675, 2014.

[20] L. O. Dissabandara, N. J. Loxton, S. R. Dias, P. R. Dodd, M. Daglish, and A. Stadlin, "Dependent heroin use and associated risky behaviour: the role of rash impulsiveness and reward sensitivity," Addictive Behaviors, vol. 39, no. 1, pp. 71-76, 2014.

[21] K. D. Ersche, P. S. Jones, G. B. Williams, A. J. Turton, T. W. Robbins, and E. T. Bullmore, "Abnormal brain structure implicated in stimulant drug addiction," Science, vol. 335, no. 6068, pp. 601-604, 2012.

[22] J. C. Stout, J. R. Busemeyer, A. Lin, S. J. Grant, and K. R. Bonson, "Cognitive modeling analysis of decision-making processes in cocaine abusers," Psychonomic Bulletin \& Review, vol. 11, no. 4, pp. 742-747, 2004.

[23] E. M. Martin, S. Dehaan, J. Vassileva, R. Gonzalez, J. Weller, and A. Bechara, "Decision making among HIV+ drug using men who have sex with men: a preliminary report from the Chicago Multicenter AIDS Cohort Study," Journal of Clinical and Experimental Neuropsychology, vol. 35, no. 6, pp. 573-583, 2013.

[24] G. M. Herek, S. Saha, and J. Burack, "Stigma and psychological distress in people with HIV/AIDS," Basic and Applied Social Psychology, vol. 35, no. 1, pp. 41-54, 2013.

[25] R. A. Lyimo, S. E. Stutterheim, H. J. Hospers, T. De Glee, A. Van Der Ven, and M. De Bruin, "Stigma, disclosure, coping, and medication adherence among people living with HIV/AIDS in northern Tanzania," AIDS Patient Care and STDs, vol. 28, no. 2, pp. 98-105, 2014.

[26] S. SeyedAlinaghi, K. Paydary, P. Afsar Kazerooni et al., "Evaluation of stigma index among people living with HIV/AIDS (PLWHA) in six cities in Iran," Thrita, vol. 2, no. 4, pp. 69-75, 2013.

[27] M.-H. Chen, T.-P. Su, T.-J. Chen, J.-Y. Cheng, H.-T. Wei, and Y.M. Bai, "Identification of psychiatric disorders among human 
immunodeficiency virus-infected individuals in Taiwan, a nineyear nationwide population-based study," AIDS Care: Psychological and Socio-medical Aspects of AIDS/HIV, vol. 24, no. 12, pp. 1543-1549, 2012.

[28] A. Pfefferbaum, M. J. Rosenbloom, S. A. Sassoon et al., "Regional brain structural dysmorphology in human immunodeficiency virus infection: effects of acquired immune deficiency syndrome, alcoholism, and age," Biological Psychiatry, vol. 72, no. 5, pp. 361-370, 2012.

[29] S. E. Varni, C. T. Miller, T. McCuin, and S. Solomon, "Disengagement and engagement coping with HIV/AIDS stigma and psychological well-being of people with HIV/AIDS," Journal of Social and Clinical Psychology, vol. 31, no. 2, pp. 123-150, 2012. 


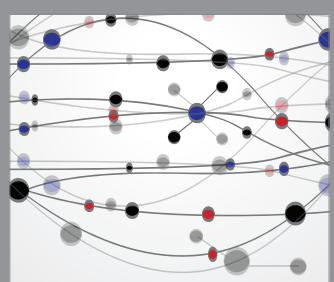

The Scientific World Journal
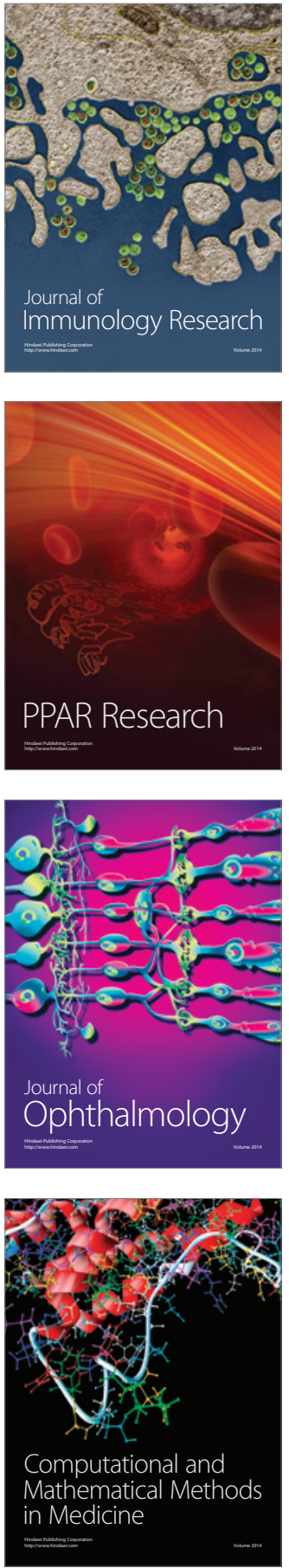

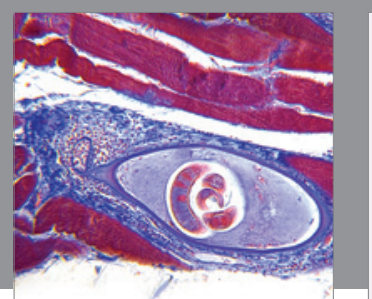

Gastroenterology Research and Practice

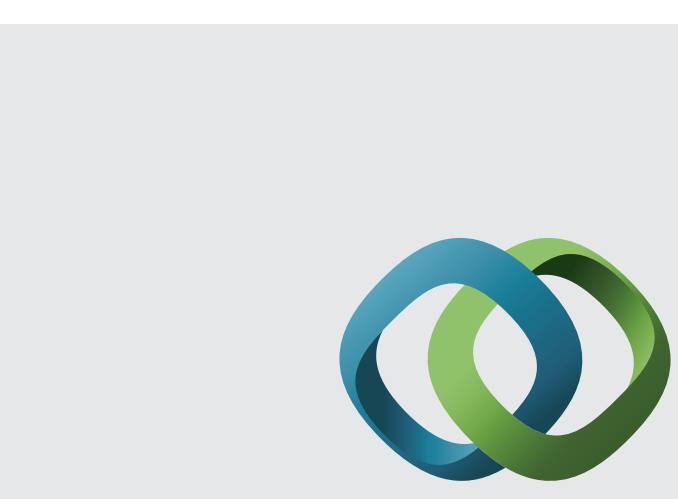

\section{Hindawi}

Submit your manuscripts at

http://www.hindawi.com
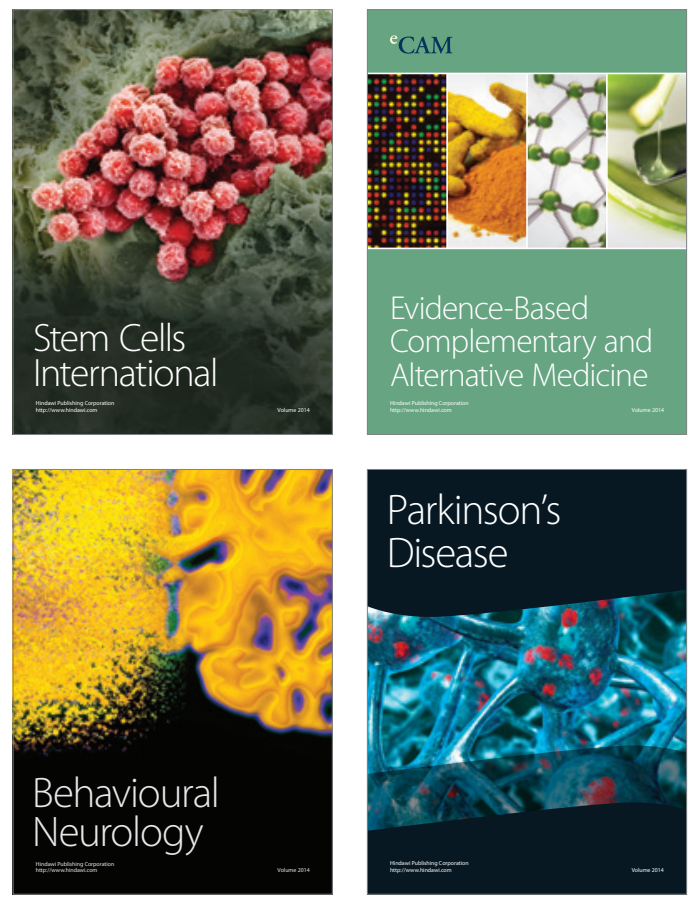
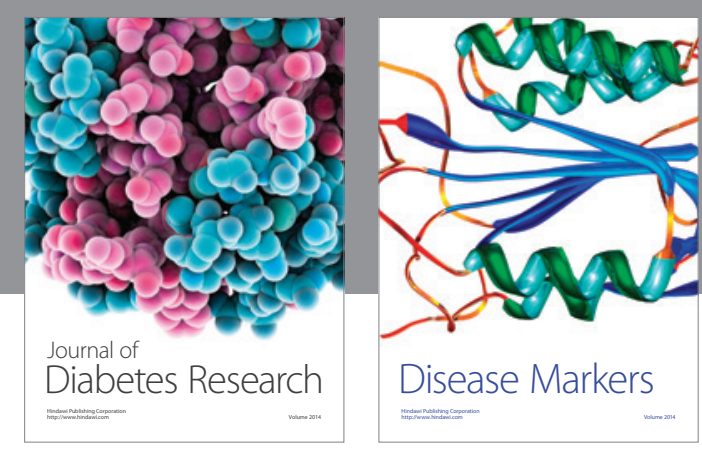

Disease Markers
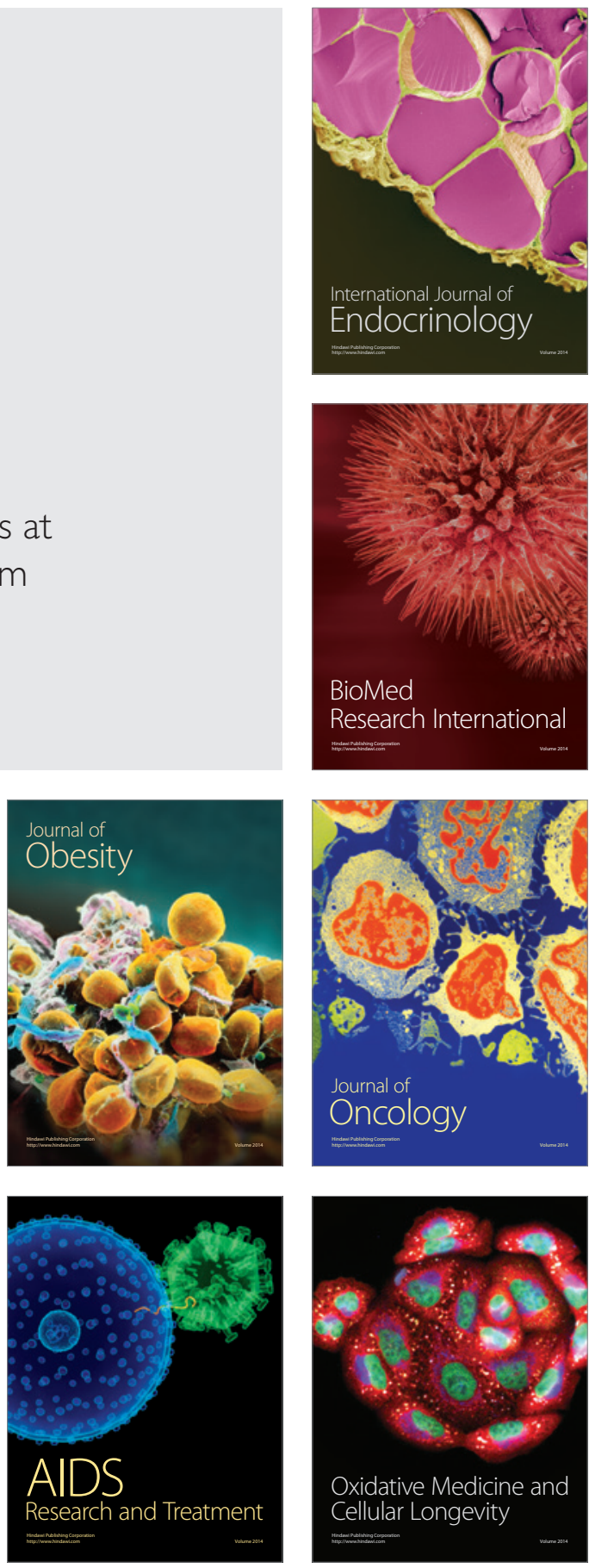